\title{
Maximum Evaporation Rates for Nonisothermal Droplets
}

\author{
F. W. HARTWIG
}

Daniel and Florence Guggenheim Jet Propulsion Center, California Institute of Technology, Pasadena, Calif.

\begin{abstract}
Calculations have been carried out in order to determine the rate of evaporation of a liquid droplet surrounded by hot gases. The present study represents an extension of earlier work by Penner on evaporation rates for isothermal droplets. In particular, allowance was made for temperature gradients within the droplet by considering a droplet composed of an isothermal core and an isothermal shell. The results obtained in the present investigation were found to be in satisfactory agreement with the known data for evaporation of isothermal droplets, thus justifying calculations for isothermal droplets as a reasonable first approximation.
\end{abstract}

\section{Introduction}

$\mathbf{T}$ HE purpose of this study is to extend earlier work on evaporation rates of isothermal droplets in rocket engines. Penner $(1)^{2}$ treated the evaporation rate of a liquid droplet in a gas at constant temperature assuming stokes flow. The limitations involved in this approximation, as well as references to the original literature, have been stated in (1) and need not be repeated here. Although the droplet temperature changed with time, it was assumed that the droplet remained isothermal during evaporation, i.e.. that the thermal conduction coefficients of evaporating liquid droplets were, for all practical purposes, infinite.

We have considered a shell model of the evaporating droplet in which the droplet is divided into two parts, an inner core and an outer spherical shell. The inner sphere was assumed to be isothermal and to remain at the original temperature of the evaporating liquid; the outer shell was also assumed to be isothermal, but its temperature was determined by making an appropriate heat balance equation. The thickness of the spherical shell was treated as a variable parameter, and it was found that results substantially equivalent to those obtained for isothermal droplets are derived, independently of the thickness of the spherical shell.

Of the evaporating droplets treated previously (1) by assuming isothermal conditions during evaporation, we have chosen for study an aniline droplet with initial radius equal to $5 \times 10^{-3} \mathrm{~cm}$. The initial temperature was set equal to $300^{\circ} \mathrm{K}$. Radiant heat transfer to the droplet was neglected since it is probably unimportant in liquid-fuel rocket motors (1).

\section{The Shell Model of the Evaporating Droplet ${ }^{3}$}

The concept of an isothermal droplet during evaporation in combustion chambers represents a first approximation. In practice one would expect that temperature gradients are set up during the life of the droplet. If the thermal conductivity is sufficiently small, then it is clear that steep tempera-

\section{Received July 26, 1952 .}

I Captain, USAF. This paper is abstracted from a thesis submitted by $F$. W. Hartwig to the graduate school of the California Fnstitute of Technology in partial fulfilment of requirements for the degree of Aeronautical Engineer, June 1952. Present address: U. S. Air Force Institute of Technologv, Wright-Patterson Air Force Base, Dayton, Ohio.

2 Number in parentheses refers to the Reference on page 243. ture gradients may be set up near the surface of the droplet. However, the surface temperature is not necessarily higher than for an isothermal droplet because it is determined by a heat balance involving cooling by evaporation.

As a first approximation to a droplet with temperature gradients, it was assumed that an evaporating droplet could be represented by an isothermal core surrounded by an isothermal shell. The shell thickness $\epsilon(\mathrm{cm})$ was set equal to $0.25,10^{-2}$, and $10^{-5}$ times the original droplet radius and was assumed to remain constant during evaporation for any given droplet.

In general the surface temperature will be determined by an energy balance between heat input into the droplet and absorption of energy by evaporation or by thermal conduction to the core of the spherical droplet. The rate of heat transfer $Q(\mathrm{cal} / \mathrm{sec})$ to a sphere of radius $r(\mathrm{~cm})$ is

$$
Q=4 \pi r^{2} h \Delta T
$$

where $\Delta T\left({ }^{\circ} \mathrm{K}\right)$ represents the temperature difference between the gases surrounding the liquid droplet and the surface temperature of the liquid droplet, and $h\left(\mathrm{cal} / \mathrm{cm}^{2} \mathrm{sec}{ }^{\circ} \mathrm{K}\right)$ is the over-all coefficient of heat transfer to a liquid droplet evaluated at the mean film temperature $T_{j}=T_{1}+{ }_{1}{ }_{2}\left(T_{g}-T_{1}\right)$. Here $T_{o}\left({ }^{\circ} \mathrm{K}\right)$ is the temperature of the hot gases, and $T_{1}\left({ }^{\circ} \mathrm{K}\right)$ is the temperature of the surface of the liquid.

For the simplified evaporation model employed for the present studies, all of the energy input occurs into a shell of volume

$V=4 / 3 \pi r^{3}-4 \pi \int_{0}^{r-\epsilon} r^{2} d r=(4 \pi / 3) r^{3}\left[1-(1-\epsilon / r)^{3}\right]$.

From Equation [2] it is seen that for $\epsilon / r \ll 1, V \simeq 4 \pi r^{2} \epsilon$; for $\epsilon / r=1$ (isothermal droplet), $V=(4 \pi / 3) r^{3}$.

It is apparent from Equations [1] and [2] that if heat transport to the spherical droplet were the only important physical process, then the temperature $T_{1}$ in the isothermal shell of thickness $\epsilon$ would rise at a rate

$$
(d T / d t)_{c}=3 h \Delta T / r\left[1-(1-\epsilon / r)^{3}\right] \rho c \ldots
$$

where $\rho(\mathrm{gm} / \mathrm{cc})$ and $c(\mathrm{cal} / \mathrm{gm})$ represent, respectively, the density and heat capacity of the liquid in the isothermal shell. For $\epsilon / r \ll 1$, Equation [3] becomes $(d T / d t)_{c}=h \Delta T / \epsilon \rho c$; for $\epsilon=1$ (isothermal droplet), $(d T / d t)_{c}=3 h \Delta T / r \rho c$.

We assumed that $\epsilon$ remains constant as the droplet radius $r$ decreases during evaporation. The rate of decrease of volume by evaporation of the isothermal shell of thickness $\epsilon$ is

$$
-d V / d t=4 \pi r^{2}(-d r / d t) \ldots
$$

The rate of absorption of heat by evaporation $Q_{e}(\mathrm{cal} / \mathrm{sec})$ is evidently

$$
Q_{e}=\left(-d V^{\%} / d t\right)(\rho l) \ldots
$$

where $l(\mathrm{cal} / \mathrm{gm})$ equals the heat of evaporation of the liquid in the shell. Since the total heat capacity of the shell is $V \rho c$, the rate of decrease of temperature in the shell, if only evaporation occurred, is

${ }^{3}$ The author is indebted to Dr. S. S. Penner for help with the formulation of the shell model of the evaporating droplet. 


$$
-(d T / d t)_{0}=(3 l / c r)(-d r / d t)\left[1-(1-\epsilon / r)^{3}\right]^{-1}
$$

The model of the evaporating droplet adopted in the present discussion requires allowance for another heat sink. Thus the assumption that $\epsilon$ is constant means that the inner surface of the isothermal shell must travel in the direction of the center of the sphere sufficiently rapidly to maintain $\epsilon$ constant. This travel of the inner surface of the outer shell means that some mass will be introduced from the colder core of the droplet into the outer shell. Since the shell must remain isothermal, heat must be added to this new mass to bring it up to the temperature of the shell. The surface area $\pm \pi(r-\epsilon)^{2}$ moves inward with a velocity $(-d r d t)$. Hence the energy absorbed per unit time in order to keep $\epsilon$ constant ind to keep the shell isothermal is

$$
\pm \pi(r-\epsilon)^{2}(-d r / d t) \rho c\left(T_{1}-T_{0}\right)
$$

In order to correct for this heat sink, we may say that of the total heat $Q$ transferred to the droplet in unit time, only the amount

$$
Q^{\prime}=4 \pi r^{2} h \Delta T-4 \pi(r-\epsilon)^{2}\left(-d r / d t \rho c\left(T_{1}-T_{0}\right) .\right.
$$

is effective in producing evaporation and heating the outer isothermal shell.

If Equation [1a] is used in place of Equation [1], Equation 3 becomes

$$
\begin{aligned}
& (d T d t)_{0}=3 h \Delta T / r\left[1-(1-\epsilon / r)^{3}\right] \rho c- \\
& \quad 3(1-\epsilon / r)^{2}(-d r / d t)\left(T_{1}-T_{0}\right) / r\left[1-(1-\epsilon / r)^{3}\right]
\end{aligned}
$$

Veglecting heat transfer between the isothermal regions, it is itmarent that

$$
(d T / d t)=(d T / d t)_{c}+\left(d T / d t_{\text {e }}\right.
$$

represents the rate of change of temperature in the isothermal shell with time. Csing Equations [3a] and [6] we obtain the result

$$
\begin{aligned}
d T / d t= & \left\{3 / r\left[1-(1-\epsilon / r)^{3}\right]\right\}\{(h \Delta T / c \rho- \\
& (1-\epsilon / r)^{2}(-d r / d t)\left(T_{1}-T_{0}\right)-(l \cdot c(-d r / d t)\}
\end{aligned}
$$

Similarly, for $\epsilon / r \ll 1$

$$
d T d t=(h \Delta T / \epsilon \rho c)-(-d r / d t)\left[(l / c \epsilon)+\left(T_{1}-T_{0}\right) / \epsilon\right]
$$

and for $\epsilon / r=1$

$$
d T / d t=3 h \Delta T / r \rho c-(3 l / c r)(-d r d t
$$

Equation [7b] represents the known result for isothermal Aroplets (1).

In order to carry out approximate calculations for the rate of change of temperature $T_{1}$ and droplet radius $r$ with time, we may assume that $-d r / d t$ is given by the Knudsen equation with an evaporation coefficient $\alpha$ set equal to unity. Thus

$$
-d r / d t=(p / \rho)(M / 2 \pi R T)^{1 / 2}
$$

where $p$ is the saturated vapor pressure of the liquid at the temperature $T_{1}, M$ is the molecular weight, and $R$ is the molar gas constant. The pair of Equations [7], [7a], or [7b] together with Equation [8] can be solved by a simple iterative procedure (1).

Representative numerical values for aniline are the following: $c=0.53 \mathrm{cal} / \mathrm{gm}^{\circ} \mathrm{K} ; r^{\circ}=5 \times 10^{-3} \mathrm{~cm} ; h=k / r=$ $4.30 \times 10^{-4} / \mathrm{r} \mathrm{cal} / \mathrm{cm}^{2} \mathrm{sec}^{\circ} \mathrm{K} ; \Delta T=\left(3000-T_{1}\right){ }^{\circ} \mathrm{K} ; \rho=$ $1.03 \mathrm{gm} / \mathrm{cc} ; l=129 \mathrm{cal} / \mathrm{gm} ;-d r / d t=\operatorname{antilog}_{10}(7.09-$ $\left.2600 / T_{1}\right) ; T_{0}=300{ }^{\circ} \mathrm{K} ; T_{r}=1650{ }^{\circ} \mathrm{K}$. The results of the present calculations for $\epsilon / r=0.25,10^{-2}$, and $10^{-4}$ are plotted in Fig. 1 together with the known results for $\epsilon / r=1$ (isothermal droplet).

Reference to Fig. 1 shows that the smaller the value of 6. $r$, the faster the shell temperature increases. However, the higher the temperature, the faster the evaporation rate.

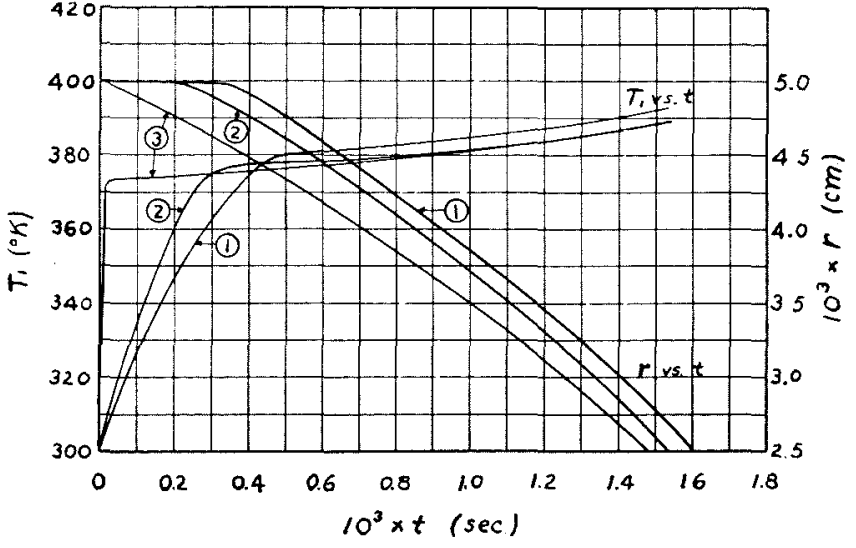

FIG. 1 DROPLET RADYUS AND SURFACE TEMPERATURE AS FUNCTIONS OF TIME FOR AN EVAPORATING DROPLET

$\begin{array}{lll}\text { (1) } \epsilon / r=1 & \text { (2) } \epsilon / r=1 / 4 & \text { (3) } \epsilon / r=10^{-2} \text { or } 10^{-5}\end{array}$

Therefore, although the temperature rises faster initially in the thinner shells, a pseudo steady-state temperature is apparently reached at a slightly lower temperature than for an isothermally evaporating droplet. In general, reference to Fig. 1. shows that the results obtained from the shell model are consistent with those obtained for the isothermal case. Hence it may be justified to conclude that calculations of evaporation rates, based on the isothermal approximation, are reasonable.

A still more realistic approximation to an evaporating droplet has been given by $\mathrm{H}$. S. Tsein who has represented the temperature profile within an evaporating droplet by a parabolic distribution. The solution of the resulting set of equations is quite laborious; 4 the formulation can be improved by utilizing a temperature profile corresponding to very large temperature gradients near the droplet surface.

${ }^{4}$ For details concerning this work, see the AE thesis of F. W. Hartwig, California Institute of Technology, June 1952.

\section{Reference}

"On Maximum Evaporation Rates for Liquid Droplets in a Rocket Motor," by S. S. Penner, Journal of The Amerrcax Rocket Society, vol. 23, March-April 1953, pp. 85-88.

\section{ARS 1953 Junior Award Competition}

Papers are now being invited for consideration for the 1953 Junior Award, to be presented at the ARS 8th Annual Convention to be held in December 1953.

The winning paper will be judged mainly on the basis of content which should reflect original thought and effort. The age of authors of papers should not exceed 25 years.

Please refer to first page, this issue of the Journal, for acceptable scope of subject matter. Manuscripts are to be prepared in accordance with style instructions on second page of this issue.

Papers must be received no later than October 1 , 1953 , and should be clearly marked, "Submitted for Junior Award Competition."

Send papers to: The Secretary, American Rocket Society, 29 West 39 th Street, New York 18, N. Y. 